\title{
Laparoscopic total extraperitoneal inguinal hernia repair
}

\section{Retrospective study on prosthetic materials, postoperative management, and quality of life}

\author{
Efi Georgiou, BSc${ }^{a}$, Elina Schoina, BSc ${ }^{a}$, Sophia-Liberty Markantonis, PhDa, Vangelis Karalis, PhDa, ${ }^{*}$, \\ Panagiotis G. Athanasopoulos, MD ${ }^{\mathrm{b}}$, Periklis Chrysoheris, MD ${ }^{\mathrm{b}}$, Fotis Antonakopoulos, $\mathrm{MD}^{\mathrm{b}}$, \\ Konstantinos Konstantinidis, $\mathrm{MD}^{\mathrm{b}}$
}

\begin{abstract}
Laparoscopic inguinal hernia repair is one of the most frequently performed operations. However, the search for the most appropriate prosthetic materials continues to occupy the surgical community. The purpose of this study was to evaluate the postoperative shortand mid-term effects (like duration of stay, number and type of complications, and inguinal pain) of laparoscopic inguinal hernia repair using the total extraperitoneal (TEP) approach. The evaluation encompassed different types of mesh and fixation devices, as well as medications prescribed during hospitalization.

This retrospective study was conducted at the General, Laparoendoscopic, Bariatric, and Robotic Surgical Clinic of the Athens Medical Center. Clinical data from 524 patients were evaluated. The answers from an appropriately designed questionnaire completed from each individual were used to obtain information about their postoperative course. The statistical analysis was implemented in SPSS v 23.

Analysis revealed that pain sensation on discharge decreased with increasing age $(P<.05)$. No clear relationship was found between surgical clips and pain $(P=.292)$, as well as mesh absorbability and chronic pain $(P=.539)$. The major postoperative complications were annoyance and discomfort (15.9\%). The recurrence rate was $1.7 \%$.

Postoperative complications following the TEP approach were mostly found to be minor; chronic pain, as an aspect of impaired quality of life, was not experienced in the majority (89.08\%). The properties of prosthetic materials used and the type of medications prescribed were not found to exert a significant role in satisfactory postoperative outcomes.
\end{abstract}

Abbreviations: $\mathrm{CCS}=$ Carolina comfort scale, $\mathrm{Gl}=$ gastrointestinal, NSAIDs = nonsteroidal anti-inflammatory drugs, TAPP = trans-abdominal preperitoneal, TEP = total extraperitoneal approach, VAS = visual analog scale.

Keywords: inguinal hernia repair, prosthetic materials, total extraperitoneal approach

\section{Introduction}

Inguinal hernia repair is one of the most common elective surgeries performed in the United States and Europe, both for adults and children, although there is great diversity among different populations. ${ }^{[1,2]}$ This type of hernia accounts for more than $70 \%$ of abdominal wall defects, while the lifetime risk for inguinal hernia is $27 \%$ in men and $3 \%$ in women. ${ }^{[3,4]}$ In general,

\footnotetext{
Editor: Johannes Mayr.

The authors have no funding and conflicts of interest to disclose.

a Department of Pharmacy, National and Kapodistrian University of Athens, Athens, ${ }^{b}$ Department of General, Laparoscopic, Robotic and Bariatric Surgery, Athens Medical Center, Marousi, Greece.

* Correspondence: Vangelis Karalis, National and Kapodistrian University of Athens, Athens, Attica, Greece (e-mail: vkaralis@pharm.uoa.gr).

Copyright (C) 2018 the Author(s). Published by Wolters Kluwer Health, Inc. This is an open access article distributed under the terms of the Creative Commons Attribution-Non Commercial License 4.0 (CCBY-NC), where it is permissible to download, share, remix, transform, and buildup the work provided it is properly cited. The work cannot be used commercially without permission from the journal.

Medicine (2018) 97:52(e13974)

Received: 3 March 2018 / Received in final form: 11 December 2018 /

Accepted: 11 December 2018

http://dx.doi.org/10.1097/MD.0000000000013974
}

inguinal hernia incidences can be divided into 2 main categories, the direct and indirect hernias, which differ in the direction at which the protrusion is apparent. In case of direct inguinal hernia, a protrusion of an organ or tissue through the inguinal canal runs medially, whereas in indirect hernia runs laterally to the inferior epigastric vessels. ${ }^{[5,6]}$

Various techniques have been used to repair inguinal hernias since the 1 st reconstructive technique described by Bassini in 1887. Today, only 3 methods are generally accepted as the best evidence-based treatment options for inguinal hernia repair: the Shouldice technique, a form of suture repair, open anterior "tension free" flat mesh repair according to Lichtenstein, and laparoscopic/endoscopic posterior flat mesh repair, principally via the transabdominal preperitoneal (TAPP) approach and the totally extraperitoneal (TEP) approach. ${ }^{[4,7,8]}$ Additionally, in recent years, the robotic approach to hernia repair has evolved as a viable/promising operative technique. ${ }^{[4]}$

Contemporary repair of hernias also requires the placement of mesh in the majority of cases. Postoperative complications and recurrences can be reduced if mechanical compatibility between the hernia meshes and the abdominal wall layers is ensured. The number of commercially available meshes and fixation devices has increased markedly in recent years. The selection of a mesh for an individual patient must take into account patient characteristics (e.g., age, size of hernia, obesity), and mesh 
properties (durability, pliability, biocompatibility, grainy texture, resistance to infection, and minimal mesh-induced foreign body responses). Currently available meshes differ with respect to their composition, structural, and mechanical parameters. ${ }^{[4]}$ Fixation devices also vary widely in terms of shape, size, and construction material. ${ }^{[9]}$ Staples are the most popular, but lately, lesstraumatic mesh fixation procedures are being used like tacks, anchor-shaped devices, and glues. ${ }^{[10]}$

Nevertheless, surgical treatment of inguinal hernia is not without complications, and in this context, the most serious midterm problems following inguinal hernia repair are recurrent hernia and chronic pain. ${ }^{[11]}$ Recent large volume systematic reviews, comparing laparoscopic repair with anterior open repair (considered as the reference technique for inguinal hernia repair), reported either no conclusive evidence of a difference in these treatment options (with respect to postoperative complications $)^{[12]}$ or benefits of the laparoscopy technique such as reduced chronic inguinal pain ${ }^{[13,14]}$ and an earlier return to normal daily activities.

The most well-known complications of the laparoscopic technique refer to urinary retention, ileus and bowel obstruction, visceral injury (small bowel, colon, bladder), and vascular injury (intra-abdominal, retroperitoneal, abdominal wall, gas embolism). ${ }^{[4]}$ A comparison of the laparoscopic approaches (TEP vs TAPP) resulted in a higher postoperative complication rate for TAPP which did not, however, result in any difference in the reoperation rate. ${ }^{[15]}$ Generally, it is expected that with the passage of time, highly experienced and dedicated hernia surgeons in large volume centers will produce more and more favorable results with TEP. Thus, the aim of this study was to evaluate the postoperative short- and mid-term effects of laparoscopic inguinal hernia repair using the TEP procedure.

\section{Materials and methods}

\subsection{Clinical unit: data collection}

This study took place at the General, Laparoendoscopic, Bariatric, and Robotic Surgical Clinic of the Athens Medical Center, after the approval by the Scientific and Ethics Committee of the hospital (KM 140667, application date of April 6, 2015). At this center, a large volume of hernia repair operations has been performed in accordance with European Hernia Society guidelines. ${ }^{[16]}$

All procedures followed were in accordance with the ethical standards of the responsible committee on the patients' anonymity and medical confidentiality. Data from a total of 524 patients who had undergone laparoscopic TEP inguinal hernia repair, using a 3-port technique, between 2010 and 2016 were included in the study. No exclusion criteria were used for patient selection.

To obtain information, pertaining to their postoperative course, patients were interviewed 10 days after surgery during their 1 st visit to the clinic following hernia repair, while for their follow-up evaluation patients were contacted by phone and asked to complete a standardized questionnaire, regarding incidence of complications, patient satisfaction, and hernia recurrence. All patients gave their informed consent for their participation in the study. All phone calls, to fill in retrospectively the questionnaire, were made between March and July 2016. This implies that the duration of the follow-up period was different for each patient ranging from few weeks up to several years after the surgery. However, this issue does not alter the validity of the results since all factors examined in our study referred to the period before, during the surgery, and few weeks after the operation which were identical for all subjects.

The data collected from the patients' medical records referred to a variety of characteristics like demographics, surgery information, and the administered medication after the operation. It was further assessed the duration of stay, the number and type of complications, and the severity of inguinal pain. Besides, special focus was placed on the evaluation of the performance of the utilized prosthetic materials like mesh and fixation devices.

The questionnaire was developed to include as much available information as possible and to be suitable for statistical analysis. In this context, all data were encoded in 3 different forms:

1. Continuous numerical variables (e.g., weight, height) were expressed in the form of the actual values (e.g., $75 \mathrm{~kg}$, etc),

2. Nominal data like symptoms (e.g., injury, relapse, type of hernia, type of mesh, etc.) were encoded either in the form of $0 / 1$ for yes/no answers, such as 0 for "no" effect (e.g., no relapse) and 1 for "yes" (e.g., if relapse was observed), or using natural numbers as for example the type of parallel hernias, if any (e.g., 1: oscheocele, 2: varicocele, 3: umbilical cord blood, 4: other)

3. Ordinal data for the cases we aimed to express a characteristic in a semi-quantitative scale (e.g., pain). In these cases, continuous physical numbers (e.g., 1, 2, 3) were used to express the severity of pain in terms of the visual analog scale (VAS).

Additional data manipulations were done if needed, depending on the findings and the aim of the analysis. For example, the VAS values for the pain on day of discharge were further grouped into 3 groups of mild, moderate, and severe pain (see "Results" section).

\subsection{Statistical analysis}

Any patients' identifiable data were transformed into anonymous information by the principal investigator of the study and statistical analysis was then applied. Initially, descriptive statistics were performed to summarize the results and get an insight into the overall performance. Depending on the type and properties (e.g., normal distribution of the data) of the variables parametric (independent and paired $t$-tests) or nonparametric methods (Mann-Whitney and Wilcoxon test) were applied. Categorical variables were compared using the Chi-squared analysis. The normality of distribution was assessed using the nonparametric Kolmogorov-Smirnov test and QQ plots. Correlation between the variables was also assessed using the Pearson or Spearman correlation coefficients. In all analyses, the significance limit was set at $5 \%$ (i.e., $P=.05$ ). The entire analysis was implemented in IBM SPSS v.23 (IBM Corp, Chicago, IL).

\section{Results}

Within a 7-year time period (from 2010 to 2016), a total of 524 patients were operated laparoscopically using 3-port TEP technique. Among them, 357 patients consented to participate in the retrospective study, 9 did not correspond, 153 were unavailable for the investigators at the time of inclusion, and 5 were deceased.

\subsection{Demographic profile}

The vast majority of patients in the study population were men, in the 5 th decade of life. Their (mean \pm standard deviation) body 


\section{Table 1}

Demographic characteristics of the 357 patients enrolled in this study.

\begin{tabular}{lc}
\hline Characteristic & Value $^{* \dagger}$ \\
\hline Gender & \\
Male & $94.40 \%(337)$ \\
Female & $5.60 \%(20)$ \\
Age, $\mathrm{yr}$ & $54.72 \pm 13.705$ \\
Weight, $\mathrm{kg}$ & $80.84 \pm 10.309$ \\
Height, $\mathrm{m}$ & $1.76 \pm 0.06986$ \\
Body mass index, $\mathrm{kg} / \mathrm{m}^{2}$ & $25.96 \pm 2.957$ \\
\hline
\end{tabular}

* The \pm values refer to the estimated standard deviation.

$\dagger$ Values in the parentheses refer to number of patients.

mass index $(25.96 \pm 2.957 \mathrm{~kg})$ indicated that overweight patients constituted a significant percentage of the sample (Table 1).

\subsection{Preoperative findings}

Preoperatively, $86 \%$ of patients complained of a bulging mass in the abdominal wall of the groin and more than $50 \%$ experienced severe pain. Other preoperative symptoms, which prompted patients to seek surgical intervention, included strangulated inguinal hernia and relapse of hernia (Table 2).

Within the patient group, unilateral, as well as bilateral inguinal hernias were found with those on the right side predominating $(43.4 \%)$. Indirect (lateral) defects were more common $(42.9 \%)$, followed by pantaloon $(42.6 \%)$ and direct $(10.1 \%)$ (Table 3$)$. A statistically significant correlation was found between gender and type of hernia $(P=.002)$.

\subsection{Mesh and fixation}

The majority of surgical mesh devices used to strengthen the hernia repair were lightweight, multifilament, and partially absorbable (Vypro; Ethicon, Machelen, Belgium) (74.8\%), and secondly the lightweight, monofilament, ultra-thin, nonabsorbable, Dipromed (Dipro Medical Devices SRL) (18.8\%) (Table 4). The mesh fixation suture materials preferred were staples (surgical clips), which were mostly nonabsorbable $(92.1 \%)$ followed by fibrin sealant $(5.1 \%)$.

\subsection{Medications}

The standardized approach to postoperative pain consisted of paracetamol and conventional nonsteroidal anti-inflammatory

\section{Table 2}

Preoperative and other symptoms of the patients' group.

\begin{tabular}{lc}
\hline & Patients $^{*}$ \\
\hline Preoperative symptom & \\
$\quad$ Inguinal pain & $56.30 \%(201)$ \\
Annoyance & $14.01 \%(50)$ \\
$\quad$ Swelling & $85.99 \%(307)$ \\
Other symptoms & \\
$\quad$ Numbness & $3.62 \%(13)$ \\
Burning sensation & $3.90 \%(14)$ \\
Heavy or dragging sensation & $2.23 \%(8)$ \\
Constipation & $1.11 \%(4)$ \\
$\quad$ Discomfort & $0.84 \%(300)$ \\
Strangulated inguinal hernia & $15.97 \%(57)$ \\
Relapse & $7.28 \%(26)$ \\
Duration of symptoms, mo & $16.65 \pm 30.029$ \\
\hline
\end{tabular}

*Values in the parentheses refer to number of patients.
Table 3

Clinical features of the performed operations.

\begin{tabular}{ll}
\hline & Patients \\
\hline $\begin{array}{l}\text { Anatomical characteristics of the hernia } \\
\text { Hernia site }\end{array}$ \\
Right & \\
Left & $43.42 \%(155)$ \\
$\quad$ Bilateral & $35.57 \%(127)$ \\
Coexistent hernias & $21.01 \%(75)$ \\
Scrotal & \\
Umbilical & $4.76 \%(17)$ \\
Varicocele & $5.32 \%(19)$ \\
Ventral & $2.52 \%(9)$ \\
Femoral & $1.12 \%(4)$ \\
Spiegel & $0.56 \%(2)$ \\
Type of hernia & $0.28 \%(1)$ \\
Direct (medial) & \\
Indirect (lateral) & $10.08 \%(36)$ \\
Pantaloon & $42.86 \%(153)$ \\
*Values in the parentheses refer to number of patients. & $42.58 \%(152)$ \\
\hline
\end{tabular}

drugs (NSAIDs), followed by opioid administration, if needed. Most frequently, patients received a combination of NSAIDs and opioids $(33.3 \%)$, followed by paracetamol-NSAIDs $(24.7 \%)$, NSAIDs alone $(14.3 \%)$, and the triple combination of NSAIDparacetamol-opioids (12.6\%). Additionally, antibiotic prophylaxis, mainly 2 nd generation cephalosporins $(82.1 \%)$, was given to patients to prevent the occurrence of postoperative infectious complications (Table 5). Proton pump inhibitors or $\mathrm{H}_{2}$-receptor antagonists were administered to prevent NSAID-associated gastrointestinal (GI) complications, in approximately a 3rd of the patients, respectively.

\section{Table 4}

Mesh and fixation devices: types and attributes.

\begin{tabular}{lc}
\hline Parameter & Patients \\
\hline Type of mesh & \\
Vypro (Ethicon) & $74.79 \%(267)$ \\
Surgi-Pro (Covidien) & $1.12 \%(4)$ \\
Dipromed & $18.77 \%(67)$ \\
Bard & $3.36 \%(12)$ \\
Ultra-Pro (Ethicon) & $0.56 \%(2)$ \\
Ti-Mesh & $0.56 \%(2)$ \\
Duzey & $0.28 \%(1)$ \\
Microval & $0.28 \%(1)$ \\
Mesh absorbability & \\
Partially absorbable & $75.35 \%(269)$ \\
Non-absorbable & $24.65 \%(88)$ \\
Type of mesh fixation & \\
Surgical clips & $92.13 \%(329)$ \\
Fibrin sealant & $5.06 \%(18)$ \\
Combination of both & $2.81 \%(10)$ \\
Type of clips & \\
Covidien (Pro-tack) & \\
Bard (Sorbafix/Absorfix, Permafix) & $41.46 \%(148)$ \\
Ethicon (Securestrap) & $50.98 \%(182)$ \\
Clips absorbability & $2.24 \%(8)$ \\
Absorbable & \\
Nonabsorbable & $38.38 \%(137)$ \\
\hline
\end{tabular}

*Values in the parentheses refer to number of patients. 
Table 5

Medications administered during hospital stay.

\begin{tabular}{lc}
\hline Medication & Patients \\
\hline Type of analgesic & \\
Paracetamol & $1.12 \%(4)$ \\
NSAIDs & $14.29 \%(51)$ \\
Opioids & $2.80 \%(10)$ \\
NSAIDs \& paracetamol & $24.65 \%(88)$ \\
Opioids \& paracetamol & $1.40 \%(5)$ \\
NSAIDs \& opioids & $33.33 \%(119)$ \\
Paracetamol \& NSAIDs \& Opioids & $12.61 \%(45)$ \\
Anticoagulants & \\
Low-molecular weight heparins & $87.68 \%(313)$ \\
Type of antibiotics & \\
Penicillins & $0.28 \%(1)$ \\
Cephalosporins (2nd generation) & $82.07 \%(293)$ \\
Quinolones & $1.68 \%(6)$ \\
Other & $0.28 \%(1)$ \\
Penicillin \& cephalosporin & $0.84 \%(3)$ \\
Penicillin \& aminoglycoside & $0.56 \%(2)$ \\
Cephalosporin \& quinolone & $0.56 \%(2)$ \\
Cephalosporin \& other & $0.56 \%(2)$ \\
Quinolone \& other & $0.28 \%(1)$ \\
Aminoglycoside \& other & $0.28 \%(1)$ \\
Muscle relaxants & $2.52 \%(9)$ \\
Gastrointestinal agents & \\
Proton pump inhibitors & $32.21 \%(115)$ \\
$\mathrm{H}_{2}$ antagonists & $31.93 \%(114)$ \\
Antiemetics & $1.68 \%(6)$ \\
Proton pump inhibitor \& antiemetic & $2.52 \%(9)$ \\
$\mathrm{H}_{2}$ antagonist \& antiemetic & $1.40 \%(5)$ \\
Proton pump inhibitor \& $\mathrm{H}_{2}$ antagonist & $1.12 \%(4)$ \\
\hline
\end{tabular}

NSAIDs = non-steroidal anti-inflammatory drugs.

* Values in the parentheses refer to number of patients.

\subsection{Postoperative course}

3.5.1. Short-term. The time from the 1 st to 10 th postoperative day was defined "short term interval." At day 10, the 1st follow-up in the clinic was scheduled. The median duration of hospital stay was 24 hours (Table 6). One of the most important short-term postoperative symptoms was pain on the day of discharge. Patients were asked to rate their pain on a VAS from 1 to 9 (1-3: mild, 4-6: moderate, 7-9: severe). For the purposes of this study, postoperative pain was alternatively categorized into groups 1, 2, 3 corresponding to no, moderate, and severe pain, respectively. Inguinal pain on discharge was characterized as mild by $76.2 \%$ of the patients and moderate by $16.9 \%$, while only a portion of $6.9 \%$ described the pain as severe (Fig. 1). Pain on discharge was found to correlate significantly with the age of patients $(P=.002$ for moderate vs no pain, $P=.05$ for moderate vs severe pain, $P<.001$ for severe vs no pain). However, no correlation was observed between pain on the day of discharge and the overall administration of analgesics $(P=.14)$ or the placement of staples $(P=.29)$. The most frequently reported short-term postoperative complications were annoyance and discomfort (15.9\%), swelling $(8.9 \%)$, seroma $(4.5 \%)$, hematoma $(3.5 \%)$, and numbness $(2.5 \%)$; however, none of them required any special treatment (Table 6).

3.5.2. Mid-term. The median time interval, from 11th postoperative day until the latest follow-up (median 1026th day or 34.2 months), was defined "mid-term interval" and ranged from 80 days to 74.7 months.
Table 6

Postoperative short-term effects.

\begin{tabular}{lc}
\hline Postoperative effect & Patients \\
\hline Duration of hospital stay & \\
$24 \mathrm{~h}$ & $91.88 \%(328)$ \\
$48 \mathrm{~h}$ & $7.28 \%(26)$ \\
$72 \mathrm{~h}$ & $0.84 \%(3)$ \\
Complications & $31.93 \%(114)$ \\
Number of complications per individual & \\
1 & $22.69 \%(81)$ \\
2 & $5.88 \%(21)$ \\
3 & $2.80 \%(10)$ \\
4 & $0.84 \%(3)$ \\
Type of complication & \\
Annoyance (discomfort) & $15.68 \%(56)$ \\
Swelling & $8.96 \%(32)$ \\
Seroma & $4.47 \%(16)$ \\
Hematoma & $3.37 \%(12)$ \\
Numbness & $2.48 \%(9)$ \\
Gl-urinary system & $1.99 \%(7)$ \\
Hypoesthesia (hyposensibility) & $1.09 \%(5)$ \\
Irritation-itching & $0.84 \%(3)$ \\
Scrotal induration & $0.56 \%(2)$ \\
Allergic reaction (inflammatory response to the mesh) & $0.28 \%(1)$ \\
Bulge (suspicion of relapse) & $0.28 \%(1)$ \\
Burning sensation (paresthesia) & $0.28 \%(1)$ \\
\hline
\end{tabular}

*Values in the parentheses refer to number of patients.

The most common mid-term complication was a feeling of annoyance or discomfort in $14.8 \%$ of patients approximately, while around $4.7 \%$ developed edema, $1.7 \%$ hypoesthesia, and $0.3 \%$ seroma in the inguinal region. Overall, $10.9 \%$ of the patients reported experiencing pain, especially during physical activity. In most patients, the pain was not severe and did not interfere with their daily living activities (Table 7). No significant association was found between the absorption characteristics of the staples $(P=.73)$ or mesh $(P=.54)$ and the development of mid-term postoperative pain. The majority of patients $(85.2 \%)$ did not mention either annoyance or discomfort. About $4.7 \%$ complained of swelling and $1.7 \%$ of numbness of the inguinal region. Another point of interest was to analyze the impact of prosthetic materials (staples, mesh, fibrin sealant) on the postoperative patients' condition. In this investigation, no significant correlation was found between the use of absorbable or nonabsorbable prosthetic materials and the total number of postoperative problems (from each individual). In particular, the significance estimates were $P=.78$ for the absorbability of staples, $P=.72$ for the absorbability of mesh, and $P=.09$ for the type of mesh fixation (staples, fibrin sealant, or combination of both). Finally, it is also worth mentioning that the total recurrence rate for laparoscopic TEP hernia repair was $1.7 \%$ (Table 8).

\section{Discussion}

The primary objective of this study was to evaluate the TEP approach to hernia repair and the types of mesh and fixation devices used during and after laparoscopic surgical repair, following the multiple-port TEP approach, in a large volume surgical unit. Also, we aimed at evaluating the postoperative management of patients and its impact on their quality of life, associated with shorter convalescence, less chronic groin pain or other complications, and decreased recurrence rates. 


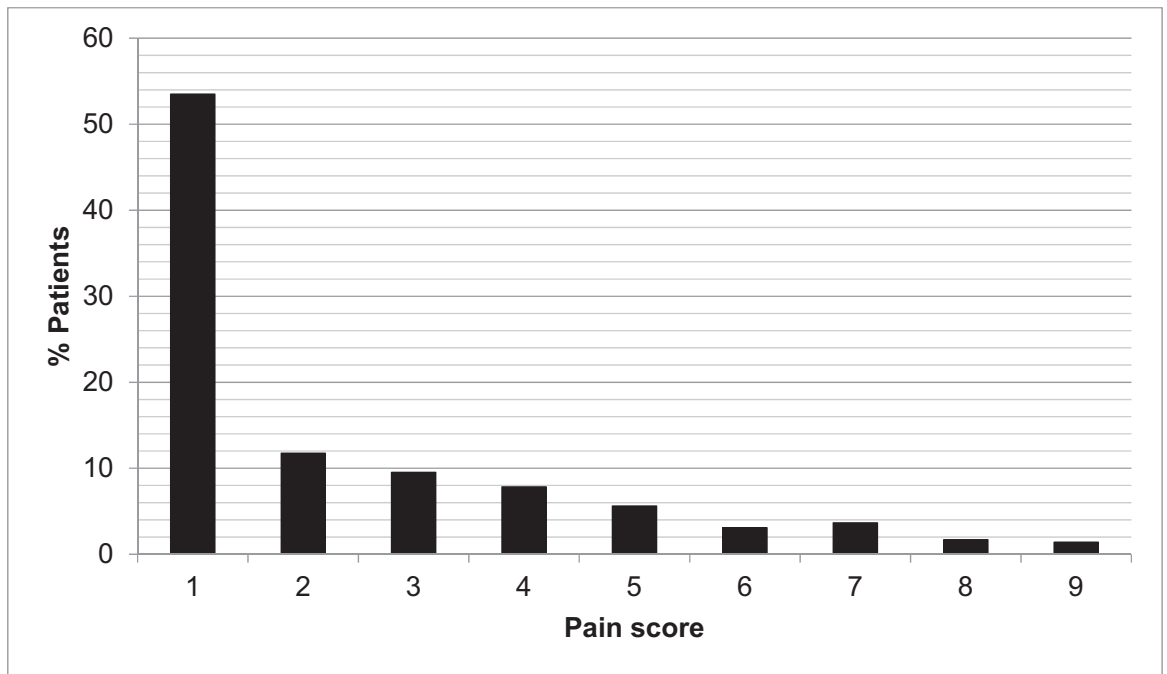

Figure 1. Percentage of patients as a function of the pain perception on the day of discharge.

\subsection{Population demographics and clinical features of inguinal hernias}

In agreement with previous studies, ${ }^{[17]}$ our study found that inguinal hernias occurred more frequently in males, aged 55 to 65 years, and were right sided and oblique (indirect). Specifically, in women indirect (lateral) hernias prevailed, whereas in men pantaloon hernias predominated followed by indirect and then direct (medial). Direct hernias appeared only in few females, because of the very narrow posterior wall of the inguinal canal. ${ }^{[18]}$ In contrast to the results of this study, Zendejas et al ${ }^{[19]}$ reported a higher frequency of direct hernias, which are followed by oblique and finally a considerably smaller number of pantaloon inguinal hernias in males (who consisted $97 \%$ of the sample).

\subsection{Intraoperative and short-term postoperative complications}

Our study found no intraoperative complications. Minor shortterm postoperative complications included annoyance and

\section{Table 7}

Postoperative mid-term effects.

\begin{tabular}{lc}
\hline Postoperative effect & Patients \\
\hline Inguinal pain & \\
At rest & $0.56 \%(2)$ \\
During physical activity & $5.32 \%(19)$ \\
Both & $5.04 \%(18)$ \\
Type of complication & \\
Annoyance (discomfort) & $14.85 \%(53)$ \\
Swelling & $0.28 \%(2)$ \\
Seroma & $4.47 \%(16)$ \\
Hematoma & $0.28 \%(2)$ \\
Numbness & $0.56 \%(2)$ \\
Gl-urinary system & $0.84 \%(3)$ \\
Hypoesthesia (hyposensibility) & $1.66 \%(6)$ \\
Irritation-itching & $0.56 \%(2)$ \\
Scrotal induration & $0.56 \%(2)$ \\
Allergic reaction (inflammatory response to the mesh) & $0.28 \%(1)$ \\
Bulge (suspicion of relapse) & $1.38 \%(5)$ \\
Burning sensation (paresthesia) & $0.83 \%(3)$ \\
\hline
\end{tabular}

$\mathrm{Gl}=$ gastrointestinal.

*Values in the parentheses refer to number of patients. discomfort, swelling, and numbness, which is completely in accordance with the literature evidence. ${ }^{[20-22]}$ About $3.5 \%$ of patients presented hematoma and $4.5 \%$ seroma. In contrast, Köckerling et $\mathrm{al}^{[15]}$ reported intraoperative complications to be $1.19 \%$. Among the short-term postoperative complications, bleeding was the most frequent $(1.15 \%)$ followed by seroma formation $(0.51 \%) \cdot{ }^{[15]}$ In the study of Bansal et al, seroma formation was noted in a significantly larger percentage of patients, that is, $32.5 \%$, followed by edema $(12.6 \%)$ and wound infection $(1.8 \%){ }^{[23]}$

\subsection{Pain on discharge}

Younger patients considered pain as severe, while older people reported little or no pain. Similar findings were reported by Nienhuijs et $\mathrm{al}^{[24]}$ who attributed the difference in pain tolerance to a reduction in number and function of peripheral nociceptive neurons and an increased pain/heat perception threshold in the elderly. Despite the differences in pain sensation no significant difference was found with the overall administration of analgesics in the present study. Preemptive, multimodal perioperative analgesia is considered another modulator of nociceptive

\section{Table 8}

Significance ( $P$-values) of all correlations considered in this study. Correlations

Variable 1

Gender

Age of patients

Type of hernia

Variable 2

$\boldsymbol{P}$

Pain on discharge

Moderate vs. no pain $\quad .002$

Moderate vs. severe $\quad .047$

Severe vs. no pain $\quad .000$

Overall administration

Pain on discharge$$
.142
$$

of analgesics

Placement of staples

Absorbability of staples

Absorbability of mesh

Absorbability of staples

Absorbability of mesh

Pain on discharge

Long-term postoperative pain 
information. ${ }^{[24]}$ A similar approach for pain management (i.e., the administration of 2 analgesics regardless of the pain assessment) was applied to our study patients and possibly this strategy may justify the lack of a statistically significant relationship between the variables. Whatsoever, the current view is that pain must be controlled before it becomes extreme and unbearable, because it puts additional stress on the body at a time when focus should be raised on wound healing. ${ }^{[25]}$

A 3rd variable having been examined, in terms of pain immediately after surgery, was the placement of staples. No statistically significant correlation was found between the 2 variables and thus, the possible idea that staples create pain was not evident $(P>.05)$ in our study.

\subsection{Mid-term post-operative complications related to hernia surgery}

The use of absorbable vs nonabsorbable clips and mesh was not found to correlate with the incidence of either mid-term pain or other postoperative problems. Also, no association was found between the use of staples, fibrin sealant, or both for prosthetic mesh fixation and the total number of postoperative problems. Mainly nonabsorbable (permanent) staples were used and the use of fibrin sealant was preferred for mesh fixation. Recent studies concur that reinforcement with fibrin sealant does not increase the risk of complications and in fact may be associated with a lower risk of complications compared with fixation techniques that penetrate tissue. ${ }^{[10,26]}$ In our study, most patients experienced no chronic pain whether the mesh used was partially absorbable or nonabsorbable $(P>.05)$. Similar findings were reported by Cristaudo et al, ${ }^{[27]}$ who compared patient comfort scores (Carolina comfort scale [CCS] that measures severity of pain, sensation, and movement limitations from implanted meshes) using the 2 mesh types and found low CCS scores that were not statistically significant. A statistically significant correlation was not noted between postoperative complications and types of meshes used, but this result was possibly skewed by the strong preference for partially absorbable meshes. It is interesting that inguinal hernia of those patients with at least one postoperative complication had been repaired more often using partially absorbable prosthetic meshes.

As already mentioned, the recurrence rate as a measure of success of inguinal hernia surgery was within the limits of the reported incidence of recurrence in TEP, that is, approximately $1 \%$ to $2 \%$. According to the published literature, known factors for the development of a recurrent inguinal hernia are surgical technique, type of hernia (direct exert higher risk than indirect), recurrent inguinal hernia (the more frequently a recurrence occurs, the higher the risk of a new recurrence), females, and smoking. ${ }^{[16,28]}$ In our study, inguinal hernia relapse was not found to be associated with any of the factors listed.

The limitations of our study included the dissimilar duration of follow-up, the relatively small size of the patients' sample and missing patient data. Thus, a statistical heterogeneity is apparent and statistical differences might not be reflected. Therefore, a well-designed prospective study with extensive follow-up is needed to explore the impact of the prosthetic materials used in laparoscopic inguinal hernia operations.

\section{Conclusion}

A statistically significant correlation was found between age and pain on the day of discharge. No intraoperative complications were demonstrated in our study group of patients. The most common mid-term complication was a feeling of annoyance or discomfort, while the recurrence rate did not exceed $1.7 \%$. TEP approach for inguinal hernia mesh repair is a feasible approach, which can yield favorable outcomes regarding postoperative complications and quality of life. Further randomized prospective studies with increased sample sizes are required to verify the findings of this analysis.

\section{Author contributions}

Conceptualization: Konstantinos M. Konstantinidis.

Data curation: Efi Georgiou, Elina Schoina, Panagiotis Athanasopoulos, Periklis Chrysoheris, Fotios Antonakopoulos, Konstantinos M. Konstantinidis.

Formal analysis: Vangelis Karalis.

Investigation: Efi Georgiou, Elina Schoina, Sophia Liberty Markantonis, Panagiotis Athanasopoulos, Periklis Chrysoheris, Fotios Antonakopoulos, Konstantinos M. Konstantinidis.

Methodology: Panagiotis Athanasopoulos, Periklis Chrysoheris, Fotios Antonakopoulos, Konstantinos M. Konstantinidis.

Project administration: Konstantinos M. Konstantinidis.

Supervision: Sophia Liberty Markantonis, Vangelis Karalis, Panagiotis Athanasopoulos, Periklis Chrysoheris, Fotios Antonakopoulos, Konstantinos M. Konstantinidis.

Writing - original draft: Efi Georgiou, Elina Schoina, Sophia Liberty Markantonis, Vangelis Karalis.

Writing - review \& editing: Efi Georgiou, Elina Schoina, Sophia Liberty Markantonis, Vangelis Karalis, Panagiotis Athanasopoulos, Periklis Chrysoheris, Fotios Antonakopoulos, Konstantinos M. Konstantinidis.

\section{References}

[1] Mizrahi H, Parker MC. Management of asymptomatic inguinal hernia: a systematic review of the evidence. Arch Surg 2012;147:277-81.

[2] Bruns NE, Glenn IC, McNinch NL, et al. Treatment of routine adolescent inguinal hernia vastly differs between pediatric surgeons and general surgeons. Surg Endosc 2016;31:912-6.

[3] LeBlanc KE, LeBlanc LL, LeBlanc KA. Inguinal hernias: diagnosis and management. Am Fam Physician 2013;87:844-8.

[4] Wagner JP, Brunicardi FC, Amid PK. Brunicardi FC, Andersen DK, Billiar TR, Dunn DL, Hunter JG, Matthews JB, Pollock RE, et al. Inguinal hernias. Schwartz's Principles of Surgery 10th edMc Grow Hill Education, New York:2015;1495-520.

[5] Castorina S, Luca T, Privitera G, et al. An evidence-based approach for laparoscopic inguinal hernia repair: lessons learned from over 1,000 repairs. Clin Anat 2012;25:687-96.

[6] Alkhateeb HM, Aljanabi TJ. A peculiar variety of indirect inguinal hernia (juxtacordal indirect inguinal hernia). Ann Med Surg 2015;4:189-92.

[7] Sajid MS, Khawaja AH, Sayegh M, et al. A systematic review comparing single-incision versus multi-incision laparoscopic surgery for inguinal hernia repair with mesh. Int J Surg 2016;29:25-35.

[8] Bittner R, Schwarz J. Inguinal hernia repair: current surgical techniques. Langenbecks Arch Surg 2012;397:271-82.

[9] LeBlanc KA, Allain BWJr, Streetman WC. Kingsnorth AN, LeBlanc KA. Laparoscopic inguinal hernia repair. Management of Abdominal Hernias 4th edSpringer, London:2013;271-84.

[10] Ceccarelli G, Casciola L, Pisanelli MC, et al. Comparing fibrin sealant with staples for mesh fixation in laparoscopic transabdominal hernia repair: a case control-study. Surg Endosc 2008;22:668-73.

[11] Nienhuijs S, Staal E, Strobbe L, et al. Chronic pain after mesh repair of inguinal hernia: a systematic review. Am J Surg 2007;194:394-400.

[12] Koning GG, Wetterslev J, van Laarhoven CJHM, et al. The totally extraperitoneal method versus Lichtenstein's technique for inguinal hernia repair: a systematic review with meta-analyses and trial sequential analyses of randomized clinical trials. PLoS One 2013;8: e52599. 
[13] Pisanu A, Podda M, Saba A, et al. Meta-analysis and review of prospective randomized trials comparing laparoscopic and Lichtenstein techniques in recurrent inguinal hernia repair. Hernia 2015;19: 355-66.

[14] Yang J, Tong DN, Yao J, et al. Laparoscopic or Lichtenstein repair for recurrent inguinal hernia: a meta-analysis of randomized controlled trials. ANZ J Surg 2013;83:312-8.

[15] Köckerling F, Bittner R, Jacob DA, et al. TEP versus TAPP: comparison of the perioperative outcome in 17,587 patients with a primary unilateral inguinal hernia. Surg Endosc 2015;29:3750-60.

[16] Simons MP, Aufenacker T, Bay-Nielsen M, et al. European Hernia Society guidelines on the treatment of inguinal hernia in adult patients. Hernia 2009;13:343-403.

[17] Berger D. Evidence-based hernia treatment in adults. Dtsch Arztebl Int 2016;113:150-7.

[18] Ellis H. Anatomy of the anterior abdominal wall and inguinal canal. Anaesth Intensive Care Med 2009;10:315-7.

[19] Zendejas B, Onkendi EO, Brahmbhatt RD, et al. Long-term outcomes of laparoscopic totally extraperitoneal inguinal hernia repairs performed by supervised surgical trainees. Am J Surg 2011;201:379-84.

[20] Birk D, Hess S, Garcia-Pardo C. Low recurrence rate and low chronic pain associated with inguinal hernia repair by laparoscopic placement of Parietex ProGrip ${ }^{\mathrm{TM}}$ mesh: Clinical outcomes of 220 hernias with mean follow-up at 23 months. Hernia 2013;17:313-20.
[21] Fumagalli Romario U, Puccetti F, Elmore U, et al. Self gripping mesh versus staple fixation in laparoscopic inguinal hernia repair: a prospective comparison. Surg Endosc 2013;27:1798-802.

[22] Swadia ND. Laparoscopic totally extra-peritoneal inguinal hernia repair: 9 year's experience. Hernia 2011;15:273-9.

[23] Bansal VK, Misra MC, Babu D, et al. A prospective, randomized comparison of long-term outcomes: chronic groin pain and quality of life following totally extraperitoneal (TEP) and transabdominal preperitoneal (TAPP) laparoscopic inguinal hernia repair. Surg Endosc 2013;27:2373-82.

[24] Nienhuijs SW, Rosman C, Strobbe LJA, et al. An overview of the features influencing pain after inguinal hernia repair. Int J Surg 2008;6:351-6.

[25] Feliciano D, Hawn M, Heneghan K. Ventral hernia repair. American College of Surgeons (ACS). Surgical Patient Education 2014;Available at: www.facs.org/patienteducation.

[26] Fortelny RH, Petter-Puchner AH, Glaser KS, et al. Use of fibrin sealant (Tisseel/Tissucol) in hernia repair: a systematic review. Surg Endosc 2012;26:1803-12.

[27] Cristaudo A, Nayak A, Martin S, et al. A prospective randomized trial comparing mesh types and fixation in totally extraperitoneal inguinal hernia repairs. Int J Surg 2015;17:79-82.

[28] Burcharth J, Pommergaard H-C, Bisgaard T, et al. Patient-related risk factors for recurrence after inguinal hernia repair: a systematic review and meta-analysis of observational studies. Surg Innov 2015;22:303-17. 